\title{
B Cell Lymphoproliferation in Spontaneously Diabetic BB Wistar Rats
}

\author{
T.A.Seemayer ${ }^{1}$, W.Schürch ${ }^{2}$ and N. Kalant ${ }^{3}$ \\ ${ }^{1}$ Department of Pathology, Montreal Children's Hospital, McGill University-Montreal Children's Hospital Research Institute, \\ ${ }^{2}$ Département de Pathologie, Hôtel-Dieu de Montréal and ${ }^{3}$ Lady Davis Institute for Medical Research, \\ Sir Mortimer B. Davis Jewish General Hospital; McGill University Faculty of Medicine, Montreal, Quebec, Canada
}

\begin{abstract}
Summary. Ninety-six spontaneously diabetic BB Wistar rats were maintained for their natural life span and, at death, were autopsied together with 86 age- and sex-matched non-diabetic BB control rats. A 15\% incidence of abdominal B cell lymphoproliferative lesions was documented in the diabetic rats compared with $1 \%$ incidence in the non-diabetic rats $(p<0.005)$. The B cell lymphoproliferative process included minute mesenteric and omental aggregates of plasma cells and small lymphocytes (one rat), atypical partially fibrotic lymphoproliferative mesenteric nodules (three rats), and malignant lymphoma with features of immunoblastic sarcoma (eight rats) or plasma
\end{abstract}

cell lymphoma (two rats). Cytoplasmic immunoglobulin was demonstrated in two of the four lymphomas examined by the peroxidase-antiperoxidase technique, thus confirming their B cell derivation. The striking incidence of $\mathrm{B}$ cell lymphoproliferation in this diabetic population is additional evidence of altered immunity in this animal model of insulin-dependent diabetes mellitus.

Key words: Diabetes mellitus, B cell lymphoma, BB Wistar rat, immunoblastic sarcoma, B cell lymphoproliferation.
The spontaneously diabetic BB Wistar rat is a recently described animal model of insulin-dependent diabetes mellitus [1]. The diabetic syndrome presents abruptly in approximately $30 \%$ of this outbred strain between 58-123 days of age (mean 88 days) [2] and is usually of severe nature requiring the daily administration of insulin to avert ketoacidosis and death. Pancreatic and blood insulin content are markedly reduced at the onset of clinical diabetes and remain so for the life of the animal.

Several lines of evidence suggest that immunological mechanisms are central to the initiation of the diabetic state. Firstly, intense lymphocytic infiltration of the pancreatic islets (insulitis) is demonstrable at the onset of clinical diabetes. This finding, the morphological hallmark of Type 1 (insulin-dependent) diabetes mellitus [3], may be produced by a variety of experimental methods, many of which induce an immunological reaction to pancreatic B cells. Secondly, both the administration of antilymphocyte serum [4] and neonatal thymectomy [5] prevent diabetes from developing in genetically susceptible $B B$ rats. Additional data which suggest that altered immunity is involved in this model include an increased percentage of circulating $B$ lymphocytes secondary to $T$ cell lymphopenia [6], chronic thyroiditis [7], and the suc- cessful passive transfer of insulitis from diabetic BB rats to nude mice [8].

If immunological mechanisms constitute a pathogenetic factor in the initiation of the syndrome, diabetic $\mathrm{BB}$ rats might be expected to exhibit a heightened incidence of lymphoproliferative lesions. Such associations are well-documented in autoimmune prone NZB [9] and $(\mathrm{NZB} \times \mathrm{NZW}) \mathrm{F}_{1}$ hybrid mice [10] and mice undergoing a chronic graft-versus-host reaction [11]. In these models immunodeficiency, defective immunoregulation, and B cell lymphoid neoplasia appear to be pathogenetically linked. Patients with genetically determined immunodeficiency and/or autoimmune disorders also bear witness to the strong relation between immunodeficiency, autoimmunity, and lymphoproliferative disorders of B lymphocytes.

This report, a more complete presentation of observations briefly annotated in 1979 [12], documents varied expressions of B cell lymphoproliferation in long-term diabetic BB rats. In contrast, non-diabetic age- and sexmatched $\mathrm{BB}$ rats were rarely affected by this process. This finding provides additional evidence of immunological disturbances in this animal model of insulin-dependent diabetes. 
Table 1. Incidence of lymphoproliferative lesions in non-diabetic and diabetic BB rats

\begin{tabular}{lr}
\hline Primary lesion & \\
Non-diabetic & 86 \\
Total examined & 1 \\
Mesenteric plasma cell lymphoma & \\
Diabetic & 96 \\
Total examined & 1 \\
Mesenteric benign plasma cell infiltration & 3 \\
Mesenteric atypical lymphoid hyperplasia & 10 \\
Mesenteric or retroperitoneal lymphoma & \\
$\quad$ Immunoblastic sarcoma (8) & \\
Plasma cell lymphoma (2) & \\
Sites of lymphomatous dissemination (diabetic) & 5 \\
Liver & 4 \\
Lymph nodes & 4 \\
Spleen & 3 \\
Lung & 2 \\
Kidney & \\
\hline
\end{tabular}

\section{Materials and Methods}

\section{Animals}

Ninety-six diabetic rats were received over the course of one year from Bio Breeding Laboratories, Ottawa, Ontario after development of diabetes as determined by glycosuria and hyperglycaemia. Eighty-six of these rats were accompanied by an age- and sex-matched non-diabetic animal from the same colony. Animals were maintained on Purina laboratory chow and water ad libitum, with daily SC injection of NPH or lente insulin (2-10 U/day), a dose individually adjusted on the basis of daily assessment of body weight, glycosuria, and ketonuria. Survival after the diagnosis of diabetes was 5-14 months (mean \pm SEM: $8^{ \pm \pm}$ 2 months) for a total life span of $12 \pm 2$ months. Death occurred spontaneously in 90 of the animals; six were sacrificed when they appeared moribund. Partial autopsy was carried out in all cases. At the time of post-mortem examination of 86 diabetic rats, an age-matched non-diabetic animal was sacrificed for comparison.

\section{Light Microscopy}

All tissues judged to represent tumour were fixed in $10 \%$ buffered formalin and processed routinely for light microscopy. Paraffin-embedded sections were stained with haematoxylin-phloxine-saffron. Selected blocks were stained by the following techniques: periodic-acidSchiff (with and without diastase digestion), methyl green pyronine, Giemsa, mucicarmine, and toluidine blue. In most but not all tumourbearing animals, sections of heart, lung, liver, spleen, lymph nodes, pancreas, and kidney were examined. Histological studies were performed on only one non-diabetic rat in which a tumour was found.

\section{Immunohistochemical Staining}

In four cases of lymphoma which showed the best cellular preservation, immunohistochemical identification and localization of immunoglobulin were studied by the peroxidase-antiperoxidase (PAP) technique described by Sternberger et al. [13]. Endogenous peroxidase activity was blocked by immersing sections in absolute methanol containing 6\% hydrogen peroxide for $30 \mathrm{~min}$. The primary antisera employed were rabbit anti-rat IgG (Miles Laboratories, Elkhart, Indiana, USA) and rabbit anti-rat immunoglobulins (Dakopatts, Cedarlane Laboratories, Hornby, Ontario, Canada), at dilutions of 1:200,1:400, and 1:800. The secondary antibody, swine anti-rabbit IgG (Dakopatts, Cedarlane Laboratories) was used at 1:20 dilution. Fresh rabbit horseradish peroxidase-antiperoxidase conjugate (Dakopatts, Cedarlane Laborato- ries), diluted 1:75, was applied for $1 \mathrm{~h}$. Bound immunoglobulin was 10 calized histochemically by a freshly prepared solution of $0.05 \% 3,3^{\prime}$-diaminobenzidine (Baker Chemicals, Philipsburg, New Jersey, USA) and $0.01 \%$ hydrogen peroxide in Tris buffer $(0.05 \mathrm{~mol} / 1, \mathrm{pH} 7.6)$. To assure that endogenous peroxidase had been blocked completely, sections were stained with the 3-3'-diaminobenzidine solution immediately after the blocking reaction and examined. Two negative controls were performed: (1) normal rabbit serum was substituted for the primary antibody and (2) the secondary antibody was omitted from the staining sequence. All sections were examined histologically; the antigen localization within neoplastic cells was determined. Positive controls were provided by paraffin sections of freshly-fixed lymph nodes from normal Wistar rats.

\section{Electron Microscopy}

Formalin-fixed tissue from the mesenteric neoplasm of two diabetic rats was processed for electron microscopy in conventional fashion.

\section{Results}

\section{Non-Diabetic Rats}

The only neoplasm identified in this group arose in the mesentery, was multilobulated, and invaded the contiguous small bowel serosa. Although somewhat autolyzed, the neoplasm was composed principally of plasma cells many of which appeared degenerate. The neoplasm was classified as plasma cell lymphoma.

\section{Diabetic Rats}

Fourteen diabetic rats had macroscopically recognizable lesions subsequently identified as lymphoproliferative in nature. In one rat, small white discolourations were scattered throughout the mesentery and omentum; in another a $1-\mathrm{cm}$ nodule was identified in the mesentery. In 12 rats a grey abdominal mass $(1-5 \mathrm{~cm}$ in length, often focally haemorrhagic) was seen in the mesentery (ten animals) or retroperitoneum (two animals).

Sections of the small white mesenteric and omental discoloured tissue revealed microscopic aggregates of mature plasma cells and small lymphocytes beneath the mesothelial surface. Multifocal infiltrates of similar nature were identified in the exocrine pancreas.

Sections from the small $(1 \mathrm{~cm})$ nodule and mesenteric mass of two rats revealed a fibrous nodule within which lymphocytes, plasma cells and large transformed pyroninophilic lymphocytes were dispersed. Many of the latter had hyperchromatic, pleomorphic nuclei some of which were cytologically atypical. This lesion was classified as atypical B lymphocyte proliferation with fibrosis. In one rat multifocal infiltrates of a similar nature were identified within the exocrine pancreas.

Sections from the ten tumour-bearing (eight mesentery, two retroperitoneum) rats disclosed a malignant lymphoma in which infiltration of contiguous pancreas, small bowel serosa, nerve trunks, autonomic ganglia, and skeletal muscle was often documented. Most tumours were focally necrotic; many were partially auto- 


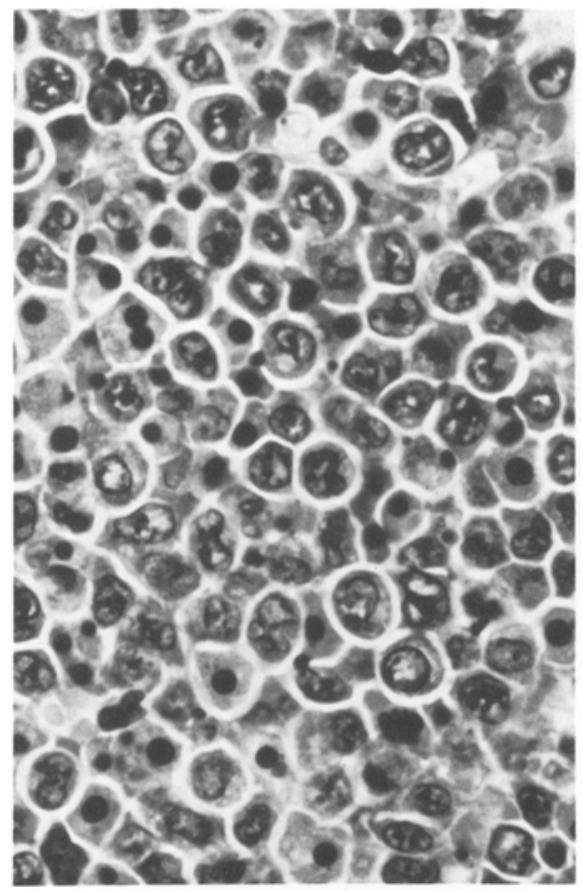

Fig. 1. Mesenteric lymphoma (immunoblastic sarcoma) composed of large transformed lymphocytes and small pyknotic degenerate-appearing plasmacytoid cells (haematoxylin-phloxine-saffron, $\times 400$ )

lyzed. Lymphomatous involvement, distinct from the abdominal mass, was documented in lymph nodes, spleen, liver, lung and kidney (Table 1).

Most lymphomas were composed of non-cohesive sheets of large cells which demonstrated variable degrees of plasmacytoid differentiation (Fig. 1). Scattered among the large lymphocytes were lesser numbers of pyknotic degenerate-appearing cells. In five lymphomas, a 'starry sky' pattern was produced, the result of phagocytosis of debris by reactive histiocytes and in three others a marked tendency to fibrosis was observed.

The neoplastic cells in eight cases were large transformed lymphocytes with round, sometimes notched nuclei, thick nuclear membranes, dense irregular chromatin, and a prominent nucleolus. Mitoses were often seen; some were abnormal. Cytoplasm was abundant, sharply defined, and slightly basophilic. Cytoplasmic pyroninophilia was demonstrated in each case although the staining intensity varied. Periodic-acid-Schiff stains (with and without diastase) were variably positive. The cytoplasm stained blue with Giemsa technique; toluidine blue stain failed to demonstrate cytoplasmic metachromasia; mucicarmine staining was negative.

Although the eight neoplasms were judged to represent immunoblastic sarcoma of B lymphocytes [14], in two the transformed lymphocytes were often multinucleated and demonstrated marked nuclear pleomorphism resembling Reed-Sternberg cells. Modest numbers of eosinophils were present as well. The histological features were similar to those of a murine lymphoma for-

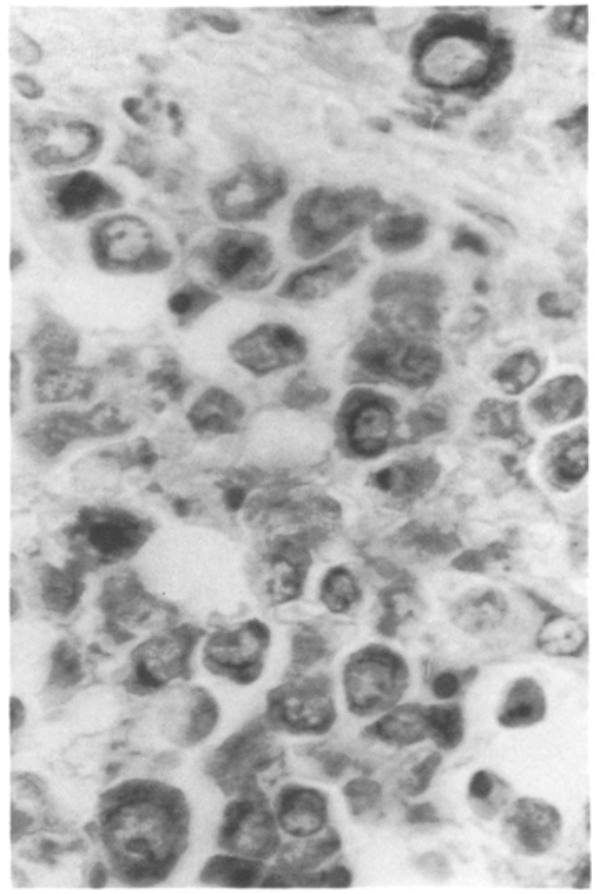

Fig. 2. Mesenteric lymphoma (immunoblastic sarcoma) demonstrating cytoplasmic granular staining reaction product (rabbit anti-rat IgG, peroxidase-antiperoxidase, $\times 600$ )

merly designated reticulum cell sarcoma, Dunn type B [15].

In the remaining two neoplasms, plasma cells comprised the dominant cell. In one, the plasma cells appeared degenerate with structureless pyknotic nuclei and acidophilic, refractile, pyroninophilic cytoplasm. In the other, numerous Russell bodies were formed. These two neoplasms were classified as plasma cell lymphomas.

In two rats with immunoblastic sarcoma, sections of mesentery remote from the main neoplasm revealed microscopic foci of nodular infiltrates of plasma cells and small lymphocytes. Similar aggregates were found in random mesenteric sections of one rat with a mesenteric nodule classified as atypical B lymphocyte proliferation with fibrosis.

Lymph node sections from normal Wistar rats examined by the peroxidase-antiperoxidase technique for both immunoglobulins and IgG, revealed modest numbers of cells with darkly stained cytoplasm in the medullary portion of the nodes. Sections stained with 3,3'-diaminobenzidine were negative, confirming that endogenous peroxidase activity had been blocked completely. Sections in which normal rabbit serum had been substituted for the primary antibody or the secondary antibody had been omitted, failed to demonstrate a reaction product. Immunoglobulins and $\mathrm{IgG}$ were demonstrated in the cytoplasm of many of the large transformed lymphocytes and plasma cells in one case of immunoblastic sarcoma (Fig. 2). Similarly, immunoglobulins and IgG were 


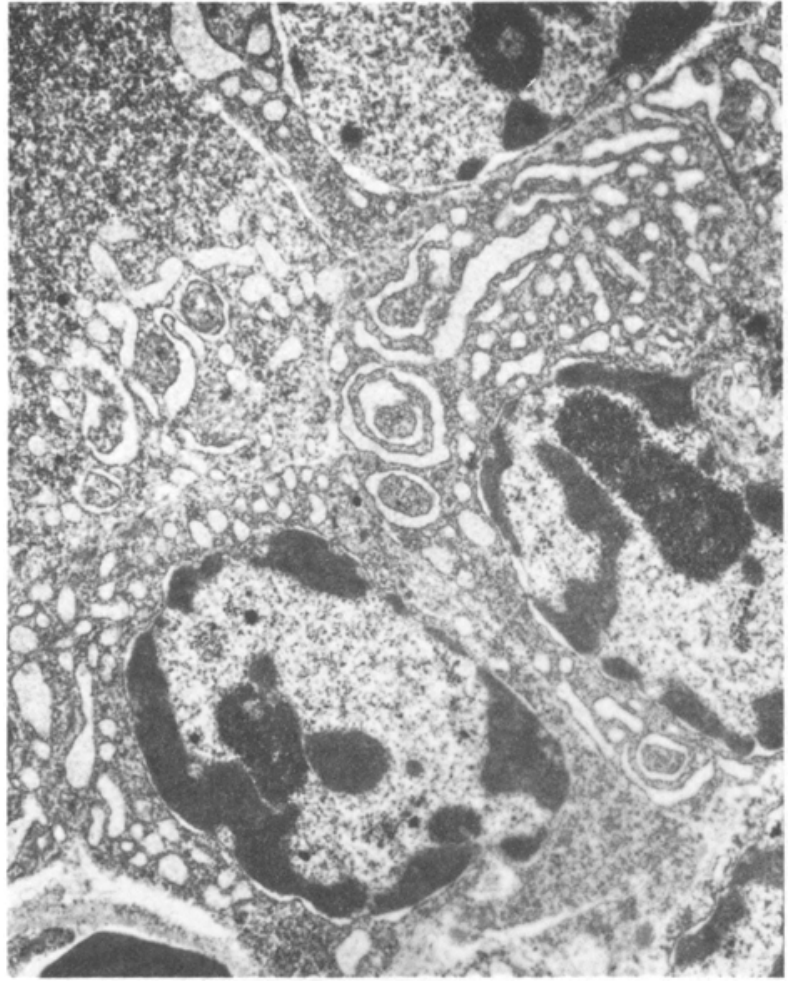

Fig. 3. Mesenteric lymphoma (immunoblastic sarcoma) demonstrating abundant, dilated rough endoplasmic reticulum and polyribosomes (transmission electron micrograph $\times 4,500$ )

demonstrated in the majority of the plasma cells in one case of plasma cell lymphoma.

Ultrastructural examination performed in two cases of immunoblastic sarcoma demonstrated large nuclei with prominent chromatin and nucleoli. The cytoplasm contained modest amounts of polyribosomes and rough endoplasmic reticulum, the latter often dilated and filled with granular material (Fig.3). The pyknotic, degenerate-appearing cells noted on light microscopy contained abundant polyribosomes and rough endoplasmic reticulum and demonstrated chromatin condensation and homogenization. These cells were judged to represent plasmacytoid lymphocytes. 'Starry sky' areas were composed of cellular debris, either extracellular or within histiocytes.

\section{Discussion}

This study describes a morphological spectrum of B cell lymphoproliferation which develops in a significant number of spontaneously diabetic BB Wistar rats maintained on insulin for 7-11 months. The age of onset of diabetes and the survival time of the affected animals did not differ significantly from those of the group as a whole. The incidence of tumours $(10 \%)$ is significant $(p$ $<0.02$ ) and the combined incidence of all lymphoproliferative lesions $(15 \%)$ is highly significant $(p<0.005)$. The $\mathrm{B}$ cell nature of the lymphoproliferative disorder is established by the plasma cell infiltrates in the benign and atypical mesenteric nodules, the pronounced plasmacytoid features of each lymphoma, the ultrastructural findings of well-developed rough endoplasmic reticulum, and the demonstration of immunoglobulins in neoplastic cells of two lymphomas. The failure to identify immunoglobulin in the two other cases in which the technique was applied is attributed to the autolysis which characterized these tumours.

The lymphoproliferative process assumes several forms ranging from minute mesenteric aggregates of small lymphocytes and mature plasma cells to fully-developed disseminated lymphoma, either immunoblastic sarcoma or plasma cell lymphoma. Occupying an intermediate, possibly linkage position, is a lesion designated atypical B lymphocyte proliferation with fibrosis. In several rats combinations of these lymphoproliferative lesions were documented, suggesting a continuum from a reactive to a neoplastic process.

The development of B cell lymphomas in the longterm diabetic BB rat is of some importance since most Wistar-derived rats tend to have a low incidence of spontaneous lymphomas. Abdominal lymphoma in Wistar rats develops in $<1 \%$ of most strains and is uncommon before 24 months of age. Even in rare Wistar-derived rats with a high incidence of lymphoma, the neoplasms tend to arise in extra abdominal sites and are uncommon before 24 months of age [16]. The association between diabetes and lymphoproliferative lesions at a relatively young age in the BB model suggests that factors pathogenetically related to the initiation of the diabetic state at 2-3 months of age, in some way, may contribute to the development of the lymphoproliferative neoplasms which emerge at a later date. Although we have not observed similar mesenteric nodular infiltrates in young diabetic BB rats (3-4 months of age), careful study of lymph nodes from such rats reveals a marked plasmacytosis (unpublished observations). These data, in the context of the present study, suggest that the B cell dependent zone of lymphoid tissue from diabetic BB rats is the site of a sustained proliferative reaction.

The diabetic $\mathrm{BB}$ rat bears strong resemblance to several murine models in which autoimmunity, immunodeficiency, defective immunoregulation, and B cell lymphoma are well documented. The best studied are the autoimmune prone NZB and $(\mathrm{NZB} \times \mathrm{NZW}) \mathrm{F}_{1}$ hybrid mice and mice undergoing a chronic graft-versushost reaction. Serial examination of lymphoid tissue in these models discloses a time-related spectrum of B cell lymphoproliferation which ranges from simple hyperplasia to atypical hyperplasia (often with pronounced plasmacytoid features) and eventual lymphoma, usually immunoblastic sarcoma [17, 18]. The lymphomas develop in a high percentage of animals and are time-dependent, i. e. directly related to age or duration of the chronic graft-versus-host reaction. Presumably the process of lymphomagenesis is contingent upon the acquisition of time-dependent specific immunoregulatory defects 
and/or specific non-random chromosomal breaks. It is possible that future studies will reveal acquired age-dependent immunoregulatory aberrations in diabetic BB rats which develop concomitant with the lymphoproliferative lesions observed in tissue.

At the present time, the spontaneously diabetic BB rat represents a promising animal model of insulin-dependent diabetes. The similarities between this model and human insulin-dependent diabetes include the pancreatic pathology, young age at onset, insulin dependence, association with the major histocompatibility complex [19], and various autoimmune associations. The emergence of lymphomas in this model constitutes a major departure from the human experience since there is no increased incidence of lymphoma in insulin-dependent diabetic patients. The factors responsible for this distinction have not been elucidated.

Acknowledgements. The assistance of Dr. N.Fukushima and $\mathrm{Mr}$. S. Chatterjee is gratefully acknowledged. Dr. R. D.C. Forbes graciously provided the primary antibodies utilized in the immunohistochemical procedure. This work was supported in part by the Medical Research Council of Canada and the McGill University - Montreal Children's Hospital Research Institute. This is publication number 82027 of the McGill University-Montreal Children's Hospital Research Institute.

\section{References}

1. Nakhooda AF, Like AA, Chappel CI, Murray FT, Marliss EB (1977) The spontaneously diabetic Wistar rat. Metabolic and morphologic studies. Diabetes 26:100-112

2. Seemayer TA, Oligny L, Tannenbaum GS, Goldman H, Colle E (1980) Animal model: spontaneous diabetes mellitus in the BB Wistar rat. Am J Pathol 101: 485-488

3. Gepts W (1965) Pathologic anatomy of the pancreas in juvenile diabetes mellitus. Diabetes 14:619-633

4. Like AA, Rossini AA, Guberski DL, Appel ML, Williams RM (1979) Spontaneous diabetes mellitus: reversal and prevention in the $\mathrm{BB} / \mathrm{W}$ rat with antiserum to rat lymphocytes. Science 206: $1421-1423$

5. Like AA, Williams RM, Kislauskis E, Rossini AA (1981) Neonatal thymectomy prevents spontaneous diabetes in the $\mathrm{BB} / \mathrm{W}$ rat. Clin Res 29: 542A (Abstract)

6. Jackson R, Rossi N, Crump T, Haynes B, Eisenbarth GS (1981) The $\mathrm{BB}$ diabetic rat. Profound T-cell lymphocytopenia. Diabetes 30 : $887-889$
7. Sternthal E, Like A, Sarantis K, Braverman L (1981) Spontaneous and iodide-induced lymphocytic thyroiditis in the diabetic biobreeding/Worcester (BB/W) rat: a model of autoimmune endocrinopathy. Program and Abstracts, 63rd Annual Meeting of the Endocrine Society, $\mathrm{p} 271$

8. Nakhooda AF, Poussier P, Sima AAF, Marliss EB (1981) Immunity in the spontaneously diabetic 'BB' Wistar rat: passive transfer of insulitis in the presence of donor rat lymphopenia. Clin Invest Med 4: 35B (Abstract)

9. Mellors RC (1966) Autoimmune disease in NZB/BI mice. II. Autoimmunity and malignant lymphoma. Blood 27:435-448

10. Greenspan JS, Gutman GA, Talal N, Weissman IL, Sugai S (1974) Thymus-antigen- and immunoglobulin-positive cells in lymphnodes, thymus, and malignant lymphomas of NZB/NZW mice. Clin Immunol Immunopathol 3:32-51

11. Schwartz RS, Beldotti L (1965) Malignant lymphomas following allogeneic disease. Transition from an immunological to a neoplastic disorder. Science 149: 1511-1514

12. Kalant N, Seemayer T (1979) Malignant lymphoma in spontaneously diabetic rats. N Engl J Med 300: 737

13. Sternberger LA, Hardy PH, Cuculis JJ, Meyer HG (1970) The unlabelled antibody enzyme method of immunohistochemistry. J Histochem Cytochem 18:315-333

14. Lukes RJ, Collins RD (1974) Immunologic characterization of human malignant lymphomas. Cancer 34: 1488-1503

15. Dunn TB, Deringer MK (1968) Reticulum cell neoplasm, type B, or the 'Hodgkin's-like' lesion of the mouse. J Nat Cancer Inst 40: $771-821$

16. Swaen GJV, Van Heerde P (1973) Tumours of the haematopoietic system. In: Turusov VS (ed) Pathology of tumours in laboratory animals, Volume 1, Part 1, Tumours of the rat, International Agency for Research on Cancer, Lyon, pp 185-200

17. Taylor CR (1976) Immuno-histological observations upon the development of reticulum cell sarcoma in the mouse. J Path 118: 201-219

18. Gleickmann E, Van Elven F, Gleickmann H (1979) Immunoblastic lymphadenopathy, systemic lupus erythematosus, and related disorders. Am J Clin Pathol 72: 708-723

19. Colle E, Guttmann RD, Seemayer TA (1981) Spontaneous diabetes mellitus in the rat. I. Association with the major histocompatibility complex. J Exp Med 154: 1237-1242

Received: 24 August 1981

and in revised form: February 11982

Dr. T. A. Seemayer

Department of Pathology

Montreal Children's Hospital

2300 Tupper Street

Montreal, Quebec H3H 1P3, Canada 